\title{
Agradecimientos
}

A todos los equipos que han participado en la producción de planta, plantaciones o riegos de apoyo: brigada de Centros de Recuperación de Fauna y Flora, brigadas Natura 2000, técnicos de los PN de Serra Calderona y Túria, y a nuestros compañeros del CIEF, Pedro Corral, Juan C. Felis y José Martí. Agradecemos asimismo a los compañeros de la Región de Murcia, en particular a Jesús Robles, por la información facilitada.

Al Ayuntamiento de Bétera y La Pobla de Vallbona, en particular a las Concejalías de Medio Ambiente, por las facilidades y terrenos aportados para las experiencias de plantación. Al IES "La Vereda" (La Pobla de Vallbona) por la colaboración prestada en la plantación de La Manguilla.

A la Comandancia Militar de Valencia y Castellón, y al personal de su Órgano de Apoyo, por la atención que ha ofrecido para la autorización y el desarrollo de las trabajos de conservación realizados dentro de la Base Militar Jaime I de Bétera, así como a la Jefatura de dicha Base y al personal del equipo coordinador de Medio Ambiente de la misma, por las facilidades prestadas y su amabilidad en todo momento.

La producción de Cistus heterophyllus subsp. carthaginensis en la Comunidad Valenciana se beneficia del soporte financiero del Fondo Europeo Agrícola de Desarrollo Rural (FEADER).

INMACULADA FERRANDO-PARDO 1,2, P. PABLO FERRER-GALLEGO 1,2, FRANCISCO J. ALBERT 1,2, VÍCTOR MARTÍNEZ1,2,

BERNAT PLANA 1,2, EMILIO LAGUNA $^{1,3}$ \& JUAN JIMÉNEZ ${ }^{3}$

1. Generalitat Valenciana. Servicio de Vida Silvestre - Centro para la Investigación y Experimentación Forestal (CIEF). Avda. Comarques del País Valencià, 114. 46930 Quart de Poblet, Valencia. E-mail: endemica_cief@gva.es 3. Generalitat Valenciana. VAERSA. Avda. de les Corts Valencianes, 20. 46015 Valencia. 2. Generalitat Valenciana. Servicio de Vida Silvestre. c/ Castán Tobeñas, 77. Ciutat administrativa 9 de octubre-Torre 1. 46018 Valencia.

Dibliografía

- Cánovas, L., J. Robles, E.B. Miras, J. López, R. Díaz, A.F. Carrillo \& L. Aznar (2018). Algunas novedade en el desarrollo de los planes de recuperación de flora de la Región de Murcia. Programa de conservación de flora silvestre amenazada de la Región de Murcia. III Congreso de Biodiversidad y Conservación de la Naturaleza: divulgación y sectores de la sociedad. 27-29 de septiembre de 2018. Universidad de Murcia.

- Ferrer-Gallego, P.P., I. Ferrando-Pardo \& E. Laguna (2015). Lusus naturae plantae in Cistus heterophyllus subsp. carthaginensis lus. obstinatus (Cistaceae). Bouteloua 21: 116-122.

- Ferrer-Gallego, P.P. I. Ferrando-Pardo, F.J. Albert, V. Martínez \& E. Laguna (2018). Obtención de material vegetal de reproducción en la Comunidad Valenciana. En: M.J. Vicente, J.J. Martínez-
Sánchez (Eds.). La jara de Cartagena (Cistus heterophyllus), una especie en peligro. Estado actual de conocimientos. Universidad Politécnica de Cartagena, Cartagena.

- Jiménez J.F P Sánchez-Gómez \& J.A Rosselló (2018). Evidencias de introgesión a partir de marcadores moleculares RAPD. En: M.J. Vicente, J.J. Martínez-Sánchez (Eds.). La jara de Cartagena (Cistus heterophyllus), una especie en peligro. Estado actual de conocimientos. Universidad Politécnica de Cartagena, Cartagena.

- Laguna E., P.P. Ferrer-Gallego, F.J. Albert \& I. Ferrando-Pardo (2018). Traslocaciones de conservación en la Comunidad Valenciana. En: M.J. Vicente, J.J. Martínez-Sánchez (Eds.). La jara de Cartagena (Cistus heterophyllus), una especie en peligro. Estado actual de conocimientos. Universidad Politécnica de Cartagena, Cartagena.
- Pawluczyk, M., J. Weiss \& M. Egea-Cortines (2018). Rastreo de eventos de hibridación de con marcadores "Código de barras". En: M.J. Vicente, J.J. Martínez-Sánchez (Eds.). La jara de Cartagena (Cis tus heterophyllus), una especie en peligro. Estado actual de conocimientos. Universidad Politécnica de Cartagena, Cartagena.

- Rosato, M., P.P. Ferrer-Gallego, C. Totta, E. Laguna \& J.A. Rosselló (2016). Latent nuclear rDNA instability in in vitro-generated plants of Cistus heterophyllus is activated after sexual reproduction with conspecific wild individuals. Botanical Journal of the Linnean Society 181: 127-137.

\section{La población de la tapenera Dol 10.15366/201822:002 de la Sierra Minera en la Región de Murcia}

Las plantas de las sierras cartageneras que corresponden al taxón descrito como Capparis zoharyi Inocencio, D. Rivera, Obón \& Alcaraz, también citadas, por confusión, como C. spinosa var. aegyptia (Lam.) Boiss. y C. aegyptia Lam. (este último es el nombre con el que se incluye en la Lista Roja de la flora vascular española desde 2008, en la categoría de amenaza EN B1ab(ii,v)+2ab(ii,v)), son arbustos de porte mayoritariamente erguido y tallos principales de tendencia erecta, que pueden alcanzar más de $2 \mathrm{~m}$ de altura, ramas lampiñas y céreas, hojas redondeadas con ápice emarginado, fuertes estípulas espinosas y flores particularmente efímeras, pequeñas y con número de estambres menor.

En la Región de Murcia se localiza el único núcleo de población conocido de la península Ibérica y de Europa continental, que se circunscribe al entorno de la ciudad de Cartagena y principalmente a la franja de sierras litorales denominadas en su conjunto Sierra Minera, donde se presentan ejemplares diseminados, por lo general en grupos de efectivos reducidos y con frecuencia junto a viejas edificaciones abandonadas, pozos y caminos mineros, especialmente en enclaves desde el Monte San Julián al Llano del Beal (localidad clásica).

En 2013, con el objetivo básico de contribuir al conocimiento de la distribución de la especie, igualmente con motivo de la presentación de una comunicación al $6^{\circ}$ Congreso de Biología de la Conservación de Plantas de la SEBiCoP, se realizó un primer seguimiento poblacional del taxón en el que se localizaron 26 poblaciones (25 nuevas o bien no publicadas con anterioridad), y se recopiló además información proveniente de otras fuentes: Dirección General de Medio Ambiente de la Región de Murcia, asociaciones ANSE y ARBA Cartagena, así como de profesionales o aficionados a la botánica. Como acción complementaria a los muestreos se herborizó un total de 50 pliegos de Capparis para el herbario de la Universidad Politécnica de Cartagena, destacando los del híbrido Capparis x innocentii López Esp. \& Alcaraz, nomen in sched. (UPCT 


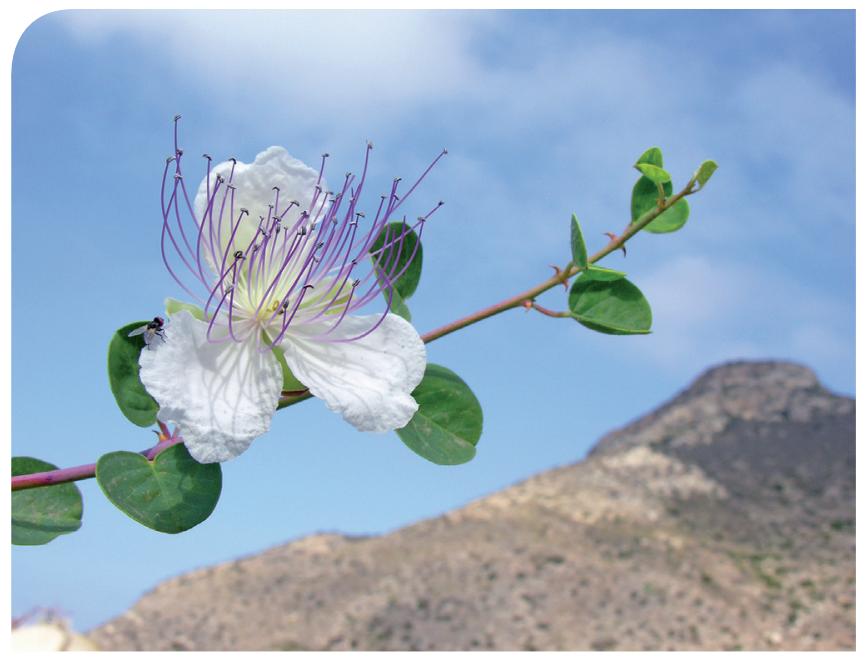

Figura 1. Rama florida de Capparis zoharyi. Al fondo, el Monte San Julián, elevación principal al este de la bocana del Puerto de Cartagena.

2990, VAL 220729), de marcados caracteres intermedios con C. sicula Veill. subsp. sicula (C. spinosa var. canescens Coss.).

En 2017, se repitió y amplió el seguimiento poblacional, confirmando hasta 44 localizaciones, presentadas en el anexo. El trabajo se centró en la caracterización de determinados grupos de individuos o subpoblaciones; además, en el mismo fueron señaladas las principales subpoblaciones para su conservación, es decir, aquellas con mayores efectivos o posición geográfica clave, delimitando de forma precisa la extensión de presencia de la planta en la Península y determinando el tamaño y dinámica de la población.

Cabe destacar de este taxón, por un lado, el paradójico desconocimiento generalizado de la planta y, por otro, su controvertida nomenclatura. En primer lugar, se da la circunstancia de que no se indica de modo alguno en obras botánicas de referencia como por ejemplo Flora iberica (Castroviejo et al. eds., 1993) o la Guía de los árboles y arbustos de la Península Ibérica y Baleares (López, 2007), lo cual ocasiona, por la repercusión e impacto de éstas como bibliografía relevante, un estatus inicial de -prácticamente- planta inexiste en España para la comunidad científica y, por ende, para el público en general. En este sentido, para el territorio español, la primera cita de esta alcaparra o tapenera se encuentra en la Lista Roja de la flora vascular española (Moreno, 2008), que procede originariamente de tres referencias de ámbito regional: de las primeras recolecciones de 1996 (MUB 48659, 48660), de la exposición pública del hallazgo mediante comunicación en formato póster en un congreso de 1997 (Alcaraz et al., 2001: 66), ambas bajo el nombre de Capparis aegyptia, y de la primera cita en el catálogo florístico de Flora de Murcia, Claves e iconografía de Plantas Vasculares (Sánchez Gómez \& Guerra, 1998), como C. spinosa var. aegyptia.

Asimismo, relacionado con lo anterior, es especialmente reseñable que, de forma reiterada, se le ha atribuido rango infraespecífico, incluso varietal, subordinado a Capparis spinosa s.l., aunque sea inequívocamente planta distinta de ésta y de $C$. aegyptia, según caracteres diagnóstico -algunos indicados al inicio- que fueron significativos para propiciar la descripción de Capparis zoharyi, como nueva especie para la Ciencia, por investigadores de la Universidad de Murcia (Inocencio et al., 2006: 145) y también de la Universidad de Jerusalén (HIBA), como Capparis hierosolymitana Danin, bi- nomen que con posterioridad se sinonimiza oportunamente a C. zoharyi para la flora de Israel.

Como se ha referido, ha sido un objetivo clave y promotor de este trabajo establecer una herramienta para la gestión y ordenación del territorio, que provea localizaciones precisas, a modo de atlas corológico, con objeto de minimizar la amenaza principal, la desaparición o transformación de su hábitat antrópico por obras, habida cuenta de que los ejemplares se presentan principalmente fuera de espacios protegidos. Al encontrarse junto a edificios mineros ruinosos y su entorno, propuestos bien de interés cultural con la categoría de sitio histórico, y márgenes viarios son, por tanto, susceptibles de actuaciones que de producirse se verán sometidas a la evaluación de la Administración pública regional, en concreto de la Dirección General de Bienes Culturales y D. G. de Carreteras. A estas también les corresponde velar por la protección de los individuos de esta especie En peligro de extinción, en cualesquiera actuaciones de su ámbito acontezcan, a través de vigilancia coordinada con el órgano ambiental, en la actualidad la Dirección General de Medio Ambiente y Mar Menor, activamente implicada en la conservación de la tapenera de la Sierra Minera, por medio de programas y seguimientos de flora protegida.

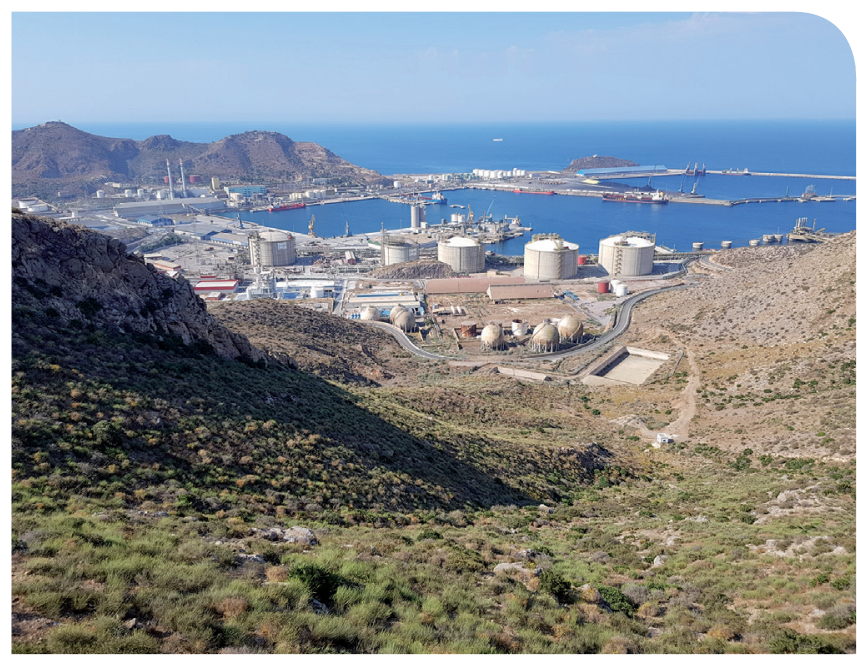

Figura 2. Ladera sur del Monte Calvario (CAL-01), desde las proximidades de la ermita de la Virgen, en cuya base se localiza el grupo con mayores efectivos de Capparis zoharyi. Al fondo, Dársena de Escombreras.

De acuerdo con el estudio realizado, en el interior de espacios protegidos quedan comprendidas 8 localizaciones (BEA02 y 03, CAR-09ABC, 10 y 11 y ATA), en el Parque Regional y Lugar de Importancia Comunitaria ES6200001 Calblanque, Monte de las Cenizas y Peña del Águila, que corresponden al $19 \%$ del total y 13 individuos, si bien son grupos viarios, en los márgenes o en el entorno inmediato de las carreteras RM-F42 y RM-314, salvo el ejemplar solitario de BEA-02.

Del mismo modo, los resultados determinan como principales grupos de individuos, primero, aquellos que perimetran el área de distribución peninsular, los que se encuentran al oeste de la bocana del Puerto de la Cartagena (POD-01 y 02, GAL), los de la autovía CT-32 km 0 (CAR-08), Los Nietos (NIE) y Cabo de Palos (PAL), en cuanto suponen avance oeste y norte de la especie. Y, fundamentalmente, los que cuentan con más de 50 ejemplares ( $9 \%$ de las localizaciones): Rambla de Alumbres (ALU-02), Llano del Beal (BEA-01), Monte El Calvario sur (CAL01) y Monte San Julián (MSJ-02), porque albergan más del $40 \%$ de los individuos peninsulares de la especie. 
Además, de los muestreos en campo se deriva un censo estimado de 575 ejemplares y la consideración de que el número de efectivos total debe ser manifiestamente inferior a 1.000 individuos, en una extensión de 149,5 ha (extensión de presencia) en la sierra de Cartagena oriental. Por tanto, el estudio de la población está en consonancia con criterio y categoría de la Lista Roja. Por otro lado, del total de localizaciones confirmadas, el 70\% corresponden a grupos con censo directo inferior a cinco individuos: evidencian una dinámica poblacional tendente a la expansión, que está produciéndose actualmente.

Finalmente, además, a esta especie rara de reducida área de distribución, por coincidencia geográfica, se le puede otorgar la distinción de especie insignia (flagship species) de la Sierra Minera de Cartagena-La Unión, territorio que -junto con Calblanque y Cabo de Palos- fue meritoriamente considerado para ser propuesto como Reserva de la Biosfera en 2008.

Por último, remarcar que, por lo expuesto, Capparis zoharyi (tapenera de la Sierra Minera) es, parafraseando a los ecólogos de la Universidad de Murcia Esteve y Miñano sobre Tetraclinis articulata (ciprés de Cartagena) en su Plan de conservación, "una de las más singulares aportaciones de la Región de Murcia a la biodiversidad en el contexto europeo".
JOSÉ ANTONIO LÓPEZ-ESPINOSA

Y FRANCISCO ALCARAZ.

Facultad de Biología, Universidad de Murcia,

Campus de Espinardo,

30100-Murcia

\section{Bibliografía}

- Alcaraz, F, C Inocencio D Rivera, F Calderón, A Robledo C Obón, A de la Torre \& C. Selma (2001). Aproximación al estudio de Capparis L. en la España peninsular y Baleares. En: F. Gómez \& J. Mota (eds.), Vegetación y Cambios Climáticos: 63-68. Universidad de Almería.

- Inocencio, C., D. Rivera, M.C. Obón, F. Alcaraz \& J.A. Barreña (2006). A systematic revision of Capparis section Capparis (Capparaceae). Annals of Missour Botanic Garden 93: 122-149.

- López Espinosa J.A. J. Sánchez Balibrea, G.G Barberá JJ Sánchez Vidal, M. Aguado, J.J. Martínez Sánchez, F. Alcaraz \& E. Diez de Revenga (2013). Apuntes sobre biología, ecología y distribución de Capparis zoharyi (tapenera de la Sierra Minera) en la Región de Murcia. Póster $6^{\circ}$ Congreso de Biología de la Conservación de Plantas de la Sociedad Española de Biología de la Conservación de Plantas en Murcia. Murcia: FloresdeMurcia.com.

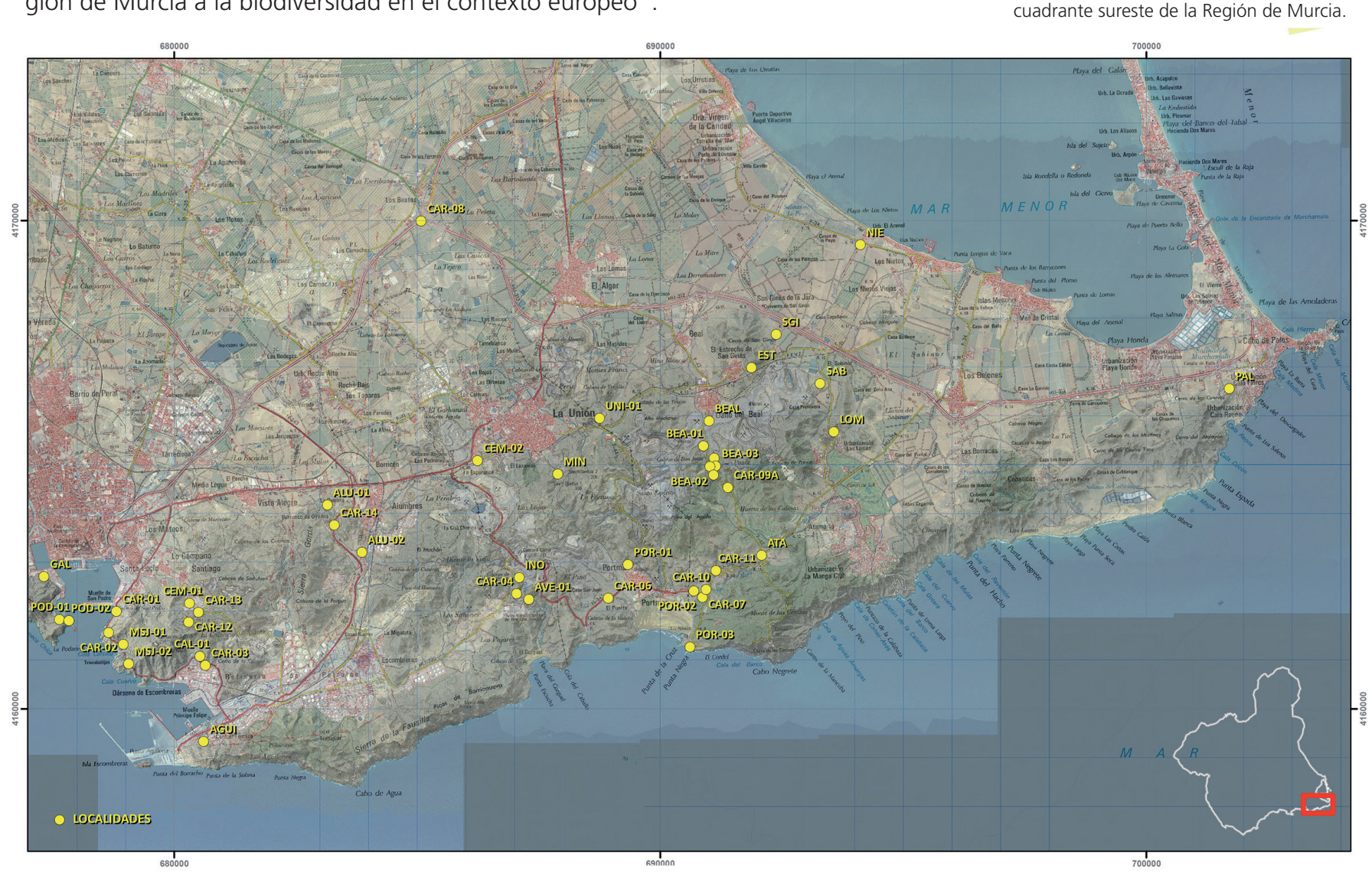

zoharyi en el área de distribución conocida de la especie en la península Ibérica, en el cuadrante sureste de la Región de Murcia.

\section{ANEXO. Coordenadas y referencias a las poblaciones conocidas de Capparis zoharyi en la Región de Murcia.}

Cartagena, Puerto de Cartagena-Galeras [GAL], 30SXG7762A, 20 m, 29-IX-2017 [1 ind.], UPCT 3056. Puerto de Cartagena-La Podadera [POD01 y 02], 30SXG7761B, 20 m, 29-IX-2017 [4 ind.]. Viaria-antigua nacional N-333 km 3 (carretera de servicio portuario Cartagena-Escombreras) [CAR-01 y 02], 30SXG7861B, 40 m, 1-VIII-2018 [7 ind.], UPCT 2859. Monte San Julián-Cala Cortina [MSJ-01], 30SXG7861D, 50 m, 30-VII2013 [4 ind.], UPCT 3029 y 3096. Monte San Julián [MSJ-02], 30SXG7860B, 30SXG7960A y 30SXG7961C, 90 m, IX-2013 [50 ind. estimados]. Cartagena-cementerio de Cartagena [CEM-01], 30SXG8062D, 60 m, 19-VII-2016 [2 ind.]. Viaria-subida a El Calvario y carretera RM-F46 km 4 [CAR-12 y 13], 30SXG8061A, 110 m, 18-VI-17 [3 ind.]. El Calvario-Monte El Calvario sur [CAL-01], 30SXG8060B y 30SXG8061D, 60 m, 30-VII-2013 [120 ind. estimados], UPCT 2860 y 2964. Viaria-antigua nacional N-333 km 6 [CAR-03], 30SXG8060B, 50 m, 3-VII-2013 [14 ind.]. Escombreras-sendero de Punta de los Aguilones [AGUI], 30SXG8059D, 30 m, 1-VIII-2018 [8 ind.]. Alumbres-La Parreta [ALU-01], 30SXG8364C, 100 m, 19-IV-2014 [12 ind.], UPCT 2698. Viaria-autovía CT-34 km 4 (antigua nacional N-343) [CAR-14], 30SXG8363B, 90 m, 5-X-2017 [2 ind.]. Alumbres-Rambla de Alumbres [ALU-02], 30SXG8363D, 50 m, 30-VII-2017 [47 ind.], UPCT 2212 y 2965. Viaria-autovía CT-32 km 0 [CAR-08], 30SXG8569A, 70 m, 5-X-2017 [4 ind.]. La Unión, La Unión-cementerio de La Unión [CEM-02], 30SXG8664A y 30SXG8865C, 120 m, 17-X2017 [4 ind.]. La Unión-Parque Minero [MIN], 30SXG8764B, 30SXG8765D y 30SXG8864A, 170 m, 24-VI-2018 [29 ind.], UPCT 3236 y 3237. Cartagena, Barranco del Avenque-Mina del Inocente [INO], 30SXG8762A, 130 m, 18-VI-2017 [7 ind.], UPCT 2211 Y 2866. Viaria-carretera 
RM-320 km 4 [CAR-04], 30SXG8762C, 140 m, 17-X-2017 [1 ind.]. La Unión, La Unión-zona minera este de La Unión [UNI-01], 30SXG8865B $150 \mathrm{~m}, 28-X-2017$ [8 ind.]. Viaria-antigua nacional N-345 km 7 [CAR-06], 30SXG8862D, 20 m, 5-X-2017 [1 ind.], UPCT 2863. Portman norte [POR-01], 30SXG8863D, 30SXG8962AB y 30SXG8963C, 40 m, 15-IX-2017 [13 ind.], UPCT 2431, 2432 y 2433. Cartagena, Llano del Beal [BEA-01], 30SXG9065ABD y 30SXG9165C, $160 \mathrm{~m}, 5-\mathrm{X}-2017$ [50 ind. estimados]. Viaria-carretera RM-314 km 1 [CAR-07 y 10] y Portman este [POR-02], 30SXG9062D, 40 m, 5-X-2017 [7 ind.], UPCT 2864. Portman-Faro de Portman [POR-03], 30SXG9061D, 40 m, 17-X-2017 [2 ind.], UPCT 2865. El Estrecho [EST], 30SXG9166B, 30SXG9266B y 30SXG9267CD, 80 m, 23-XI-2017 [7 ind.]. Llano del Beal pueblo [BEAL], 30SXG9165A, 130 m, 5-X-2017 [2 ind.]. Viaria-carretera RM-F42 km 4 [CAR-09C], 30SXG9165C, 200 m, 5-X-2017 [1 ind.]. Llano del Beal-Collado de los Mosquitos [BEA-02] y Mina Brígida [BEA-03] y viaria-carretera RM-F42 km 5 y 4 [CAR-09AB], 30SXG9164A, 210 m, 5-X-2017 [4 ind.], UPCT 3250. Viaria-carretera RM-314 km 1 [CAR-11], 30SXG9162A, 80 m, 5-X-2017 [2 ind.] Cerro de San Ginés [SGI], 30 SXG9267A, 160 m, 13-VII-2013 [1 ind.]. Viaria-Atamaría (carretera RM-314 km 3) [ATA], 30SXG9263C, $140 \mathrm{~m}, 12-X-2012$ [3 ind.], UPCT 2196. El Sabinar [SAB], 30SXG9366A, 80 m, VII-2013 [3 ind.]. Campo de Golf La Manga Club-Las Lomas [LOM], 30SXG9365B, 160 m, 10-X-2013 [23 ind.], UPCT 2961. Los Nietos-Rambla de Ponce [NIE], 30SXG9469A, 5 m, 15-IX-2017 [1 ind.]. Cabo de Palos-cantera Cala Reona, 30SYG0166B, 60 m, $4-X-2017$ [23 ind.]

\section{Plant for the Planet: un proyecto en el que los jóvenes y niños somos los protagonistas Do1: 10.15366/12018.22.003}

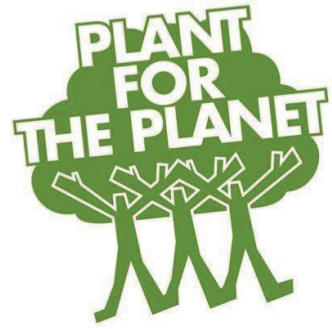

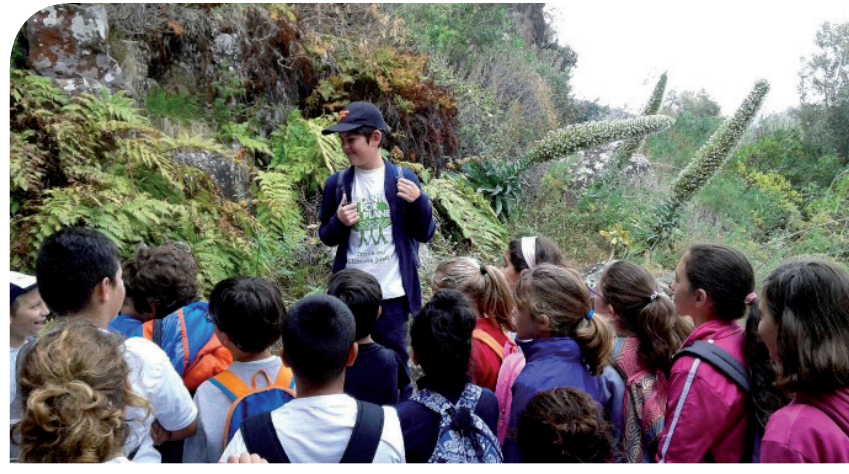

Figura 1. Joaquim durante una actividad de PFTP en el Jardín Canario.

En el año 2007 Félix Finkbeiner -con tan sólo 9 años- creó Plant for the Planet (PFTP), un proyecto internacional gracias al que se han plantado más de 15 millones de árboles con la participación de 180 países. Hoy, Félix tiene 20 años y ha iniciado The Trillion Tree Campaign, una gran campaña de reforestación a nivel mundial, demostrando que juntos -los jóvenes- podemos hacer mucho para cuidar el planeta. Esta iniciativa cuenta con el apoyo de Naciones Unidas.

Todo empezó cuando Félix, preparando una exposición sobre los osos polares para sus compañeros del colegio, descubrió que el descongelamiento de los polos estaba amenazando la supervivencia de los osos, y también la de los humanos. Inspirado por Wangari Maathai, Premio Nobel de la Paz, que impulsó el movimiento "Cinturón Verde" con el que se plantaron 30 millones de árboles en África, Félix pensó que el método más eficaz para combatir el cambio climático era plantando árboles. Los árboles tienen la capacidad de absorber grandes cantidades de $\mathrm{CO}_{2}$, que cuando se acumula en exceso en la atmósfera provoca el calentamiento global del planeta. Además, Félix se dio cuenta de que los niños y jóvenes son los que, durante más tiempo y más acusadamente, sufriremos las consecuencias de la crisis climática y por eso decidió pasar a la acción. Actualmente, hay 75.000 embajadores de PFTP en todo el mundo, unos 2.900 en España.

Tuve la suerte de conocer a Félix Finkbeiner cuando presentó PFTP en Gran Canaria, y desde entonces he tratado de promover actividades y de impulsar la "Red de embajadores" en las Islas Canarias, pues se sabe que las consecuencias del cambio climático van a afectar más a las islas pequeñas que a otras zonas del planeta. Aunque algunos se empeñen en negarlo, hay datos científicos claros sobre el incremento de las temperaturas y la mayor frecuencia de fenómenos climáticos extremos. Gracias a PFTP soy más consciente de la injusticia climática que sufren algunos países (contaminan poco pero como dependen de lo que cultivan, los cambios en el clima les afectan más). También sé, por ejemplo, que hay predicciones para 2050 que apuntan a que, si no impedimos que las temperaturas sigan en aumento, Gran Canaria, Fuerteventura y Lanzarote, además de gran parte de la península Ibérica, se transformarán en desiertos.

Me gusta formar parte de PFTP porque me siento bien sabiendo que estoy aportando algo positivo al planeta. Por eso, es importante que más jóvenes y niños se unan a PFTP, ya que si no hacemos nada, estos cambios en el clima van a ir a peor, afectando a la supervivencia de todas las especies del planeta, incluida la nuestra.

Desde que en 2014 se creó la Fundación Plant for the Planet-España se están realizando en diferentes ciudades "academias de PFTP", en las que se aprende sobre cambio climático y se hacen plantaciones. En otoño están previstas nuevas academias y, además tendremos un stand del proyecto en las miniferias de las Semanas de la Ciencia organizadas en Gran Canaria. También del 23 al 25 de noviembre de este año se va a convocar a los embajadores de PFTP de toda España en un encuentro en Barcelona para compartir ideas, hacer propuestas y unir fuerzas para lograr nuestros principales objetivos: concienciar a otros jóvenes y niños sobre las consecuencias del cambio climático y plantar el mayor número posible de árboles.

Si quieres saber más del proyecto visítanos en nuestra web https://www.plant-for-the-planet.org y también puedes apoyarnos siguiendo la campaña que hemos puesto en marcha los embajadores de PFTP en Twitter: \#PftPGreenThinking. 